\title{
CHAOTIC MAPS WITH RATIONAL ZETA FUNCTION
}

\author{
H. E. NUSSE
}

\begin{abstract}
Fix a nontrivial interval $X \subset \mathbf{R}$ and let $f \in C^{1}(X, X)$ be a chaotic mapping. We denote by $A_{\infty}(f)$ the set of points whose orbits do not converge to a (one-sided) asymptotically stable periodic orbit of $f$ or to a subset of the absorbing boundary of $X$ for $f$.

A. We assume that $f$ satisfies the following conditions: (1) the set of asymptotically stable periodic points for $f$ is compact (an empty set is allowed), and (2) $A_{\infty}(f)$ is compact, $f$ is expanding on $A_{\infty}(f)$. Then we can associate a matrix $A_{f}$ with entries either zero or one to the mapping $f$ such that the number of periodic points for $f$ with period $n$ is equal to the trace of the matrix $\left[A_{f}\right]^{n}$; furthermore the zeta function of $f$ is rational having the eigenvalues of $A_{f}$ as poles.

B. We assume that $f \in C^{3}(X, X)$ such that: (1) the Schwarzian derivative of $f$ is negative, and (2) the closure of $A_{\infty}(f)$ is compact and $f^{\prime}(x) \neq 0$ for all $x$ in the closure of $A_{\infty}(f)$. Then we obtain the same result as in A.
\end{abstract}

1. Introduction and statement of the results. This paper deals with an analysis of the periodic points for a continuous mapping from a nontrivial interval into itself. We refer the reader to $\S 2$ for the definitions.

The question "Given that a continuous mapping $f$ from the real line into itself has a periodic orbit with period $n$, for some fixed positive integer $n$, which other positive integers must occur as periods of the periodic orbits of $f$ ?" has been discussed and has been answered by Li and Yorke [7], Sharkovsky [14], Stefan [17], Straffin [18], and Block [3].

The main result is due to Sharkovsky (see Stefan $[\mathbf{1 7}]$ ). Let the positive integers be lexicographically ordered in the following way: $3 \dashv 5 \dashv 7 \dashv \ldots \dashv 2.3 \dashv 2.5 \dashv$ $2.7 \dashv \cdots \dashv 2^{2} .3 \dashv 2^{2} .5 \dashv 2^{2} .7 \dashv \cdots \dashv 2^{3} \dashv 2^{2} \dashv 2 \dashv 1$. Sharkovsky's theorem says the following. Let $f$ be a continuous mapping from $\mathbf{R}$ into itself, which has a periodic orbit of periodic $n$. Then $f$ has a period orbit of period $m$ for every $m \in \mathbf{N}$ for which $n \dashv m$ holds.

One may ask the following question: "Given any continuous mapping $f$ from a nontrivial interval into itself. Fix any positive integer $n$. How many periodic points with period $n$ does $f$ have?" Smale and Williams [16] have answered this question for the mapping $f:[0,1] \rightarrow[0,1]$ defined by $f(x)=3.83 x(1-x)$.

Received by the editors May 1, 1984 .

1980 Mathematics Subject Classification (1985 Revision). Primary 26A18, 58F 13, 58F15, 58F20.

Key words and phrases. Iteration of mappings, periodic points, semigroup of chaotic mappings, zeta function.

Research supported in part by Netherlands Organization for the Advancement of Pure Research (Z.W.O), the State University of Utrecht, and National Science Foundation Grant MCS 8120790. 
Assume that the periodic points for a continuous mapping $f$ are isolated. Then following Artin and Mazur [2], the zeta function $\zeta_{f}$ of $f$ is defined by

$$
\varsigma_{f}(z)=\exp \sum_{n=1}^{\infty}\left[\frac{N(\operatorname{Per}(f ; n))}{n} z^{n}\right]
$$

with $z$ a complex variable and $N(\operatorname{Per}(f ; n))$ the number of periodic points for $f$ with period $n$. The following question arises: "What kind of function is $\zeta_{f}$ ?"

Our first result is the following theorem.

THEOREM A. Let $f$ be a chaotic $C^{1}$-mapping from a nontrivial interval $X$ into itself. Assume that $f$ satisfies the following conditions:

(1) the set of asymptotically stable periodic points for $f$ is compact (an empty set is allowed);

(2) the set of points, whose orbits do not converge to an asymptotically stable periodic orbit of $f$, or to a subset of the absorbing boundary of $X$ for $f$, is a (nonempty) compact set, and $f$ is expanding on this set.

Then, for some well-defined square matrix $A_{f}$ with entries either one or zero, we have:

(i) the number of periodic points for $f$ with period $n$ is equal to the trace of the matrix $\left[A_{f}\right]^{n}$ for each positive integer $n$;

(ii) the zeta function $\zeta_{f}$ of $f$ is a rational function with the property that $\zeta_{f}$ has a pole in $z=\lambda^{-1}$ of order the multiplicity of $\lambda$, where $\lambda$ is an eigenvalue of the matrix $A_{f}$.

Singer [15] introduced the Schwarzian derivative for real valued mappings (see also Allwright [1]. For any mapping $f$ of class $C^{3}$, the Schwarzian derivative of $f$ at a point $x$ with $f^{\prime}(x) \neq 0$, denoted by $S f(x)$, is defined by $S f(x)=f^{\prime \prime \prime}(x) / f^{\prime}(x)$ $3 / 2 \cdot\left[f^{\prime \prime}(x) / f^{\prime}(x)\right]^{2}$.

Singer's main result can be formulated as follows (see also Misiurewicz [9], Collet and Eckmann [5], and Preston [13]): Let $f$ be any mapping of class $C^{3}$ from the unit interval $[0,1]$ into itself for which the following conditions hold: (1) $f$ has finitely many critical points, i.e. points at which the derivative of $f$ vanishes, and (2) $f$ has a negative Schwarzian derivative. Assume that $f$ has an asymptotically stable periodic point $p$ such that the direct domain of attraction of $p$ does not contain a boundary point of the unit interval. Then there is a critical point $c$ of $f$ such that the orbit of $c$ converges to the orbit of $p$.

Consequently, for the well-studied one-parameter family of maps $\left\{f_{a}\right\}$ with $f_{a}$ defined by $f_{a}(x)=a x(1-x)$ on the unit interval and parameter $a$ in the interval $[1,4]$, the conditions $(1)$ " $f_{a}$ has a (one-sided) asymptotically stable periodic orbit" and (2) "the orbit of the critical point of $f_{a}$ converges to a (one-sided) asymptotically stable periodic orbit of $f_{a}$ " are equivalent.

Now we will state our second result.

THEOREM B. Let $f$ be a chaotic $C^{3}$-mapping from a nontrivial interval into itself. Assume that $f$ satisfies the following conditions:

(1) $f$ has a negative Schwarzian derivative;

(2) the set of points whose orbits do not converge to an asymptotically stable periodic orbit of $f$, or to a one-sided asymptotically stable periodic orbit of $f$ or to 
a subset of the absorbing boundary of the interval, is contained in some compact subset of the interval, and this compact set does not contain critical points of $f$.

Then for some well-defined square matrix $A_{f}$, with entries either zero or one, we have that the conclusions (i) and (ii) in Theorem A hold.

COROLLARY B.1. Let $f$ be a chaotic $C^{3}$-mapping from a nontrivial compact interval into itself with finitely many critical points. Assume that $f$ satisfies the conditions:

(1) $f$ has a negative Schwarzian derivative;

(2) the orbit of each critical point of $f$ converges to an asymptotically stable periodic orbit of $f$, or to a one-sided asymptotically stable periodic oribit of $f$.

Then we have: $\zeta_{f}$ is a rational function.

COROLlARY B.2. Let $f$ be a chaotic polynomial mapping from a nontrivial compact interval into itself for which each critical point is real.

If the orbit of each critical point of $f$ converges to an asymptotically stable periodic orbit of $f$, or to a one-sided asymptotically stable periodic orbit of $f$, then we have: $\zeta_{f}$ is a rational function.

REMARKS. (1) If a continuous mapping from a nontrivial interval into itself has finitely many periodic points, then it is easy to show that the zeta function is rational. Furthermore, by Sharkovsky's theorem, there exists $N \in \mathbf{N}$ such that each periodic point has period $2^{N}$.

(2) Let $f$ be a chaotic $C^{1}$-mapping from a compact interval $X$ into itself. Assume that $f$ satisfies the conditions (1) the set of asymptotically stable periodic points for $f$ is compact, and (2) the set of points whose orbits do not converge to an asymptotically stable periodic orbit or to a one-sided asymptotically stable periodic orbit is a compact set.

Then $\zeta_{f}$ is not necessarily a rational function (cf. Theorem A). However, if $\left|f^{\prime}\right|^{-1 / 2}$ is a strictly convex function on intervals on which $f^{\prime}$ has no zeros, and if the orbit of each critical point of $f$ converges to an asymptotically stable periodic orbit of $f$ or to a one-sided asymptotically stable periodic orbit of $f$, then we have that $\zeta_{f}$ is a rational function. (If $f$ is a $C^{3}$-function then the conditions $\left|f^{\prime}\right|^{-1 / 2}$ is strictly convex and $f$ has a negative Schwarzian derivative are equivalent (see Allwright [1] and also Preston [13]). Hence the assertion follows from Corollary B.1.)

(3) The unpublished paper by Milnor and Thurston [8] gave algebraic procedures for counting the number of "monotone equivalence classes" of periodic orbits for continuous maps with finitely many turning points (see also Collet and Eckmann [5], Nitecki [11], and Preston [13]). Hence the results in this paper for maps with finitely many critical points and e.g. with negative Schwarzian derivative would follow from the paper by Milnor and Thurston cited. But even in this case the proofs given in this paper are different and more direct.

(4) In this paper the results are obtained for differentiable mappings, but they also hold for continuous maps with property $\mathrm{R}$. For maps with property $\mathrm{R}$ the reader is referred to Allwright [1] (see also Preston [13]).

The organization of the paper is as follows. In $\S 2$ we present all the preliminary definitions and notations. $\S 3$ deals with a semigroup of continuous mappings; to each mapping we associate a matrix with nonnegative integers as entries. We give 
the main lemmas for the proof of Theorem $\mathrm{A}$ in $\S 4$; some lemmas for the proof of Theorem $B$ are presented in $\S 5$. Finally in $\S 6$ we prove the results.

ACKNOWLEDGMENT. This paper, written during a stay at MSRI, Berkeley, and revised in Groningen is based on a part of the author's thesis [12] at the University of Utrecht, supervised by Hans Duistermaat. I would like to thank him for the stimulating and critical remarks. It is pleasure to thank Larry Castro and Wilma van Nieuwamerongen for the typing of the manuscripts. The referee's comments are gratefully ackowledged.

2. Preliminary definitions and notations. Throughout the paper $X \subset \mathbf{R}$ will be nontrivial interval. Let $E$ and $F$ be nonempty subsets of $X$ such that $E \subset F$. We write $\mathrm{Cl}(F)$ for the closure of $F, \operatorname{Bd}(F)$ for the boundary of $F$, and $\operatorname{Int}(F)$ for the interior of $F$. We denote the complement of $E$ in $F$ by $F \backslash E$.

Let $f: X \rightarrow X$ be a continuous mapping. For any $n \in \mathbf{N}$, the $n$th iterate of $f$, denoted by $f^{n}$, is inductively defined by $f^{n}=f \circ f^{n-1}$ with $f^{0}$ the identity mapping. The image of $E$ under $f^{n}$, denoted by $f^{n}(E)$, is the set $\left\{f^{n}(x) ; x \in E\right\}$; the pre-image of $E$ under $f^{n}$, denoted by $f^{-n}(E)$ or by $\left(f^{n}\right)^{-1}(E)$, is the set $\left\{x \in X ; f^{n}(x) \in E\right\}$. The set $E$ is called positively $f$-invariant if $f(E) \subset E, E$ is called negatively $f$-invariant if $f^{-1}(E) \subset E$, and $E$ is called (completely) $f$ invariant if $f(E) \subset E$ and $f^{-1}(E) \subset E$. The set $E$ is called a component of $F$ if it is a maximally connected subset of $F$; a component is called trivial if it consists of one point.

For any point $x \in X$ the orbit of $x$ under $f$ is the set $\left\{f^{n}(x) ; n \in \mathbf{N} \cup\{0\}\right\}$. A point $x \in X$ is called a periodic point for $f$ with period $p$, for some $p \in \mathbf{N}$, if $f^{p}(x)=x$ (i.e. if $x$ is a fixed point of the mapping $f^{p}$ ); the period is called primitive if it is the smallest one.

A point $x \in X$ is called a nonwandering point for $f$ if for every open neighborhood $U$ of $x$ there exists $n \in \mathbf{N}$ such that $f^{n}(U) \cap U \neq \varnothing$.

A fixed point $x$ of $f$ is called asymptotically stable if there exists an open neighborhood $U(x)$ of $x$ in such that $\lim _{n \rightarrow \infty} f^{n}(y)=x$ for all $y \in U(x)$. A point $x \in X$ is called an asymptotically stable periodic point for $f$ with period $p$ if $x$ is an asymptotically stable fixed point of $f^{p}$. The orbit of an asymptotically stable periodic point for $f$ is called an asymptotically stable periodic orbit of $f$. A periodic point $x$ for $f$ is called unstable if it is not asymptotically stable.

A periodic point $x$ for $f$ with period $p$ in the interior of $X$ is called onesided asymptotically stable if there exists a positive real number $\varepsilon$ such that either $\lim _{n \rightarrow \infty} f^{n p}(y)=x$ for all $y \in\left[x, x+\varepsilon\left[\right.\right.$ and $\left|f^{p}(y)-x\right|>|y-x|$ for all $y \in] x-\varepsilon, x\left[\right.$, or $\lim _{n \rightarrow \infty} f^{n p}(y)=x$ for all $\left.\left.y \in\right] x-\varepsilon, x\right]$ and $\left|f^{p}(y)-x\right|>|y-x|$ for all $y \in] x, x+\varepsilon[$.

A point $x \in \mathbf{R} \cup\{+\infty\} \cup\{-\infty\}, x \notin X$, is called an absorbing boundary point of $X$ for $f$ with period $p$ (in fact $p=1$ or $p=2$ ) if there exists an open set $U \subset X$ such that $f^{p k}(y) \rightarrow x$ when $k \rightarrow \infty$ for all $y \in U$. Note that an absorbing boundary point cannot be a periodic point for $f$. For clarity we will give an example. Consider the well-studied one-parameter family of maps $f_{\alpha}: \mathbf{R} \rightarrow \mathbf{R}$ defined by $f_{\alpha}(x)=\alpha x(1-x)$, with $\alpha>0$. The fixed point $x=0$ is asymptotically stable if and only if $0<\alpha<1$, the fixed point $x=0$ is one-sided asymptotically stable if and only if $\alpha=1$, and the point $-\infty$ is an absorbing boundary point for $f$ if and only if $\alpha \geq 1$. 
Assume that $q \in X$ is an asymptotically stable fixed point of $f$. The domain of attraction of $q$ is the set of points whose orbits converge to $q$; this set is open in $X$. The direct domain of attraction of $q$ is the component of the domain of attraction of $q$ containing $q$.

Assume that $q$ is an absorbing boundary point of $X$ for $f$ with primitive period $p$. The set $\left\{y \in X ; f^{p k}(y) \rightarrow q\right.$ when $\left.k \rightarrow \infty\right\}$ is called the domain of attraction of $q$; the component of this set containing $q$ as a boundary point is called the direct domain of attraction of $q$.

A point $x \in X$ is called an asymptotically periodic point for $f$ if $\lim _{n \rightarrow \infty} f^{n m}(x)$ exists for some $m \in \mathbf{N}$. A point $x \in X$ is called aperiodic if the following two conditions are satisfied: (1) $x$ is not an asymptotically periodic point and (2) the orbit of $x$ is bounded. The mapping $f$ is called chaotic if there exists a least one aperiodic point for $f$.

From now on we assume that $f \in C^{1}(X, X)$. A point $x \in X$ is called a critical point for $f$ if $f^{\prime}(x)=0$. Let $Y \subset X$ be a closed positively $f$-invariant set. The mapping $f$ is called expanding on $Y$ if we can find a constant $c>0$ and a constant $K>1$ such that $\left|\left(f^{n}\right)^{\prime}(x)\right| \geq c \cdot K^{n}$ for all $x \in Y$ and for each $n \in \mathbf{N}$.

If $f$ is of class $C^{3}$, then the Schwarzian derivative of $f$, denoted by $S f$, is defined by $S f(x)=f^{\prime \prime \prime}(x) / f^{\prime}(x)-3 / 2 \times\left[f^{\prime \prime}(x) / f^{\prime}(x)\right]^{2}$ for all $x \in X$ with $f^{\prime}(x) \neq 0$.

We write

$\operatorname{Per}(f): \quad$ the set of periodic points for $f$,

$\operatorname{Per}(f ; n)$ : the set of periodic points for $f$ with period $n$,

$\Omega(f): \quad$ the set of nonwandering points for $f$,

Crit $(f)$ : the set of critical points for $f$.

We write the symbol $\sqcup$ for disjoint union, and we use the symbol $\square$ to indicate the end of a proof.

Finally,

2-1. Let $D_{0}$ be the union of the direct domains of attraction of all (one-sided) asymptotically stable periodic points for $f$ and the absorbing boundary points of $X$ for $f$. We write $A_{0}$ for $X$ and we write $A_{1}$ for the complement of $D_{0}$ in $X$. We define by induction $A_{k+1}=\left\{x \in A_{k} ; f^{k}(x) \in A_{1}\right\}$ for each positive integer $k$. For each $k \in \mathbf{N}$ we define $D_{k}=\left\{x \in A_{k} ; f^{k}(x) \in D_{0}\right\}$. Further we set $A_{\infty}(f)=\bigcap_{k=0}^{\infty} A_{k}$.

2-2. Lemma. $A_{0}=A_{k+1} \sqcup \bigsqcup_{j=0}^{k} D_{j}$ for each $k \in \mathrm{N} \cup\{0\}$.

Proof. Fix any nonnegative integer $k$. Then $A_{k+1}=\left\{x \in A_{k} ; f^{k}(x) \in A_{1}\right\}=$ $\left\{x \in A_{0} ; f^{j}(x) \in A_{1}\right.$ for all $\left.j, 0 \leq j \leq k\right\}$, i.e. $A_{k+1}$ is the set of points which will not be mapped into $D_{0}$ under the map $f^{k}, D_{k}=\left\{x \in A_{k} ; f^{k}(x) \in D_{0}\right\}=\{x \in$ $A_{0} ; f^{j}(x) \in A_{1}$ for all $\left.j, 0 \leq j \leq k-1, f^{k}(x) \in D_{0}\right\}$, i.e. $D_{k}$ is the set of points in $A_{k}$ that will be mapped into $D_{0}$ under the map $f^{k}$. Obviously, the intersection of $A_{k+1}$ with $\bigcup_{j=0}^{k} D_{j}$ is empty. Splitting the set $A_{0}$ in the following way,

$$
A_{0}=\left\{x \in A_{0} ; f^{k}(x) \in A_{1}\right\} \cup\left\{x \in A_{0} ; f^{k}(x) \in D_{0}\right\},
$$

we get

$$
A_{0}=A_{k+1} \cup D_{0} \cup \bigcup_{j=1}^{k}\left\{x \in A_{0} ; f^{i}(x) \in A_{1}, 0 \leq i \leq j-1, f^{j}(x) \in D_{0}\right\} .
$$


We conclude $A_{0}=A_{k+1} \cup \bigcup_{j=0}^{k} D_{j}$.

REMARK. We assume from now on that $f$ is a chaotic mapping.

3. Semigroup of maps. Let $C$ denote an open set in $X$ consisting of finitely many, say $N(C)$, disjoint components. Accordingly, we write $C=\bigsqcup_{i=1}^{N(C)} C_{i}$. We denote by $\mathfrak{A}(C)$ the class of continuous functions $g: X \rightarrow X$ for which the following conditions hold:

3-1. (i) $C$ is a negatively $g$-invariant set;

(ii) the restriction of $g$ to $g^{-1}(C)$ is locally a homeomorphism;

(iii) the set $g^{-1}(C)$ has finitely many components.

3-2. Lemma. If $g, h \in \mathfrak{A}(C)$, then $g \circ h \in \mathfrak{A}(C)$.

Proof. We assume that $g, h \in \mathfrak{A}(C)$. Then we have

(i) $(g \circ h)^{-1}(C)=h^{-1}\left(g^{-1}(C)\right) \subset h^{-1}(C) \subset C$;

(ii) the restriction of $g \circ h$ to $(g \circ h)^{-1}(C)$ is locally a homeomorphism, since

$$
\left.(g \circ h)\right|_{(g \circ h)^{-1}(C)}=\left.\left.g\right|_{\operatorname{Im}\left(\left.h\right|_{(g \circ h)^{-1}(C)}\right)} \circ h\right|_{(g \circ h)^{-1}(C)} ;
$$

(iii) for every component $D$ of $g^{-1}(C)$, the number of components of the set $h^{-1}(D)$ is not larger than the number of components of the set $h^{-1}(C)$. Hence the set $(g \circ h)^{-1}(C)$ has finitely many components.

\section{3-3. COROLlaRY. If $g \in \mathfrak{A}(C)$, then $g^{n} \in \mathfrak{A}(C)$ for each $n \in \mathbf{N}$.}

ProOF. By induction.

For $g \in \mathfrak{A}(C)$ we introduce a matrix $A_{C ; g}$ with the entry $A_{C ; g}(i, j)$ equal to the number of components of the set $g^{-1}\left(C_{i}\right) \cap C_{j}, 1 \leq i, j \leq N(C)$. Such a matrix will be called a matrix associated to the pair $(C ; g)$.

\section{3-4. Lemma. If $g, h \in \mathfrak{A}(C)$, then $A_{C ; g \circ h}=A_{C ; g} \cdot A_{C ; h}$.}

ProOF. Assume that $g, h \in \mathfrak{A}(C)$. Choose integers $i, k ; 1 \leq i, k \leq N(C)$.

For a component $D$ of the set $h^{-1}\left(g^{-1}\left(C_{i}\right)\right) \cap C_{k}$ we have $g \circ h(D)=C_{i}$. From this it follows that $h(D)$ is a component of the set $g^{-1}\left(C_{i}\right)$; consequently $h(D)$ is a component of the set $g^{-1}\left(C_{i}\right) \cap C_{j}$ for some integer $j, 1 \leq j \leq N(C)$.

For a connected set $E \subset C_{j}$ we have, by the assumption $h_{i}^{-1}(C) \subset C$ and the assumption that the restriction of $h$ to $h^{-1}(C)$ is locally a homeomorphism, the property:

$$
\begin{aligned}
& \operatorname{Card}\left\{F ; F \text { a component of the set } h^{-1}(E) \cap C_{k}\right\} \\
& \quad=\operatorname{Card}\left\{F ; F \text { a component of the set } h^{-1}\left(C_{j}\right) \cap C_{k}\right\}=A_{C ; h}(j, k) .
\end{aligned}
$$

In other words, the number of the components of the set $h^{-1}(E) \cap C_{k}$ is equal to the number of components of the set $h^{-1}\left(C_{j}\right) \cap C_{k}$, this number is the entry $A_{C ; h}(j, k)$ of the matrix $A_{C ; h}$. 
Computation of the entry $A_{C ; g \circ h}(i, k)$ gives

$$
\begin{aligned}
A_{C ; g \circ h}(i, k)= & \left.\operatorname{Card}\left\{D ; D \text { a component of } h^{-1}\left(g^{-1} C_{i}\right)\right) \cap C_{k}\right\} \\
= & \sum_{j=1}^{N(C)} \operatorname{Card}\left\{D ; D \text { a component of } h^{-1}\left(g^{-1}\left(C_{i}\right)\right) \cap C_{k},\right. \\
\left.h(D) \text { a component of } g^{-1}\left(C_{i}\right) \cap C_{j}\right\} & \\
= & \sum_{j=1}^{N(C)} \operatorname{Card}\left\{E ; E \text { a component of } g^{-1}\left(C_{i}\right) \cap C_{j}\right\} \\
& \cdot \operatorname{Card}\left\{F ; F \text { a component of } h^{-1}(E) \cap C_{k},\right. \\
= & \sum_{j=1}^{N(C)} \operatorname{Card}\left\{E ; E \text { a component of } g^{-1}\left(C_{i}\right) \cap C_{j}\right\} \\
& \cdot \operatorname{Card}\left\{F ; F \text { a component of } h^{-1}\left(C_{j}\right) \cap C_{k}\right\} \\
= & \sum_{j=1}^{N(C)} A_{C ; g}(i, j) \cdot A_{C ; h}(j, k) .
\end{aligned}
$$

We conclude: $A_{C ; g \circ h}=A_{C ; g} \cdot A_{C ; h}$.

3-5. Corollary. If $g \in \mathfrak{A}(C)$, then $A_{C ; g^{n}}=\left[A_{C ; g}\right]^{n}$ for each $n \in \mathbf{N}$.

PROOF. By induction.

REMARK. $\mathfrak{A}(C)$ is a semigroup. Let $\mathfrak{m}$ denote the semigroup consisting of square matrices of size $N(C)$, with nonnegative integers as entries. The mapping $\phi: \mathfrak{A}(C) \rightarrow \mathfrak{m}$ defined by $\phi(g)=A_{C ; g}$ is a homomorphism.

3-6. LEMMA. Assume that $g \in \mathfrak{A}(C)$. Let $U \subset C$ be an open set with the properties: (1) $g^{-1}(U) \subset U$, and (2) each component of $U$ is a component of $g^{-1}(C)$. Then we have:

(i) $g \in \mathfrak{U}(U)$;

(ii) the entries of the matrix $A_{U ; g}$ are either zero or one.

ProOF. We assume that $g \in \mathfrak{A}(C)$. Let $U \subset C$ be an open set as in the lemma.

(i) Since $g^{-1}(U) \subset U \subset g^{-1}(C)$, we have that the restriction of $g$ to $g^{-1}(U)$ is locally a homeomorphism. Further we know that the set $g^{-1}(U)$ has at most $\sum_{k=1}^{N(C)} \sum_{i=1}^{N(C)} A_{C ; g^{2}}(i, k)$ components. We conclude that $g \in \mathfrak{A}(U)$.

(ii) For each component $D$ of $U$ we have that $\left.g\right|_{D}: D \rightarrow g(D)$ is a homeomorphism.

3-7. COROllary. For $g \in \mathfrak{A}(C)$ we have $g \in \mathfrak{A}\left(g^{-1}(C)\right)$ and the matrix $A_{g^{-1}(C) ; g}$ is an $N\left(g^{-1}(C)\right) \times N\left(g^{-1}(C)\right)$ matrix with entries either zero or one.

In some sense inspired by Bowen's work [4], we give the following definition.

3-8. DEFINITION A continuous mapping $g: X \rightarrow X$ has a Markov partition if there exist at most finitely many nontrivial closed intervals, say $\left\{U_{j} ; 1 \leq j \leq K\right\}$ 
and $\left\{V_{k} ; 1 \leq k \leq L\right\}$ for some positive integer $K$ and $L$, such that:

(i) $\Omega(g) \subset\left[\bigcup_{j=1}^{K} \operatorname{Int}\left(U_{j}\right) \cup \bigcup_{k=1}^{L} \operatorname{Int}\left(V_{k}\right)\right]$, in other words, the nonwandering set of $g$ is contained in the union of the interiors of the intervals.

(ii) $U_{i} \cap U_{j}=\varnothing$ for all $i \neq j, 1 \leq i, j \leq K$, and $\operatorname{Int}\left(U_{i}\right) \cap \operatorname{Int}\left(V_{k}\right)=\varnothing$ for every $1 \leq i \leq K, 1 \leq k \leq L$.

(iii) $g\left[\bigcup_{j=1}^{K} U_{j}\right] \supset \bigcup_{j=1}^{K} U_{j}, g\left[\bigcup_{k=1}^{L} V_{k}\right] \subset \bigcup_{k=1}^{L} V_{k}$.

3-9. LEMMA. Fix any $g \in \mathfrak{A}(C)$. Assume that $g$ has no one-sided asymptotically stable periodic points. If the complement of the union of the domains of attraction $A_{\infty}(f)$ is in $C$ and if the intersection of the set of asymptotically stable periodic points with the closure of $C$ is empty, then $g$ has a Markov partition.

ProOF. Fix any mapping $g \in \mathfrak{A}(C)$ such that $A_{\infty}(g) \subset C$ and the set of asymptotically stable periodic points is contained in the interior of $X \backslash C$. Choose the union of the closure of the components of the set $g^{-1}(C)$. If the set of asymptotically stable periodic points for $g$ is not empty, choose the components of the set $X \backslash C$ which contain an asymptotically stable periodic point for $g$. The chosen sets of components satisfy the conditions of Definition 3-8. We conclude that $g$ has a Markov partition.

We fix a mapping $g \in \mathfrak{A}(C)$ which has a Markov partition, say $B$. We set $\mathcal{U}=B \cap g^{-1}(B)$. It is obvious that $\mathcal{U}$ is also a Markov partition for $g$. We assume that $\mathcal{U}$ has $N(\mathcal{U})$ disjoint components. Accordingly, we write $\mathcal{U}=\bigcup_{k=1}^{N(\mathcal{U})} I_{k}$, where $I_{k}$ is a component of $\mathcal{U}, 1 \leq k \leq N(\mathcal{U})$.

In a similar manner as before, we can associate a matrix $A_{u ; g}$ to the pair $(\mathcal{U} ; g)$. From the definition of the Markov partition $\mathcal{U}$ and Corollary 3-7 it follows that the matrix $A_{u ; g}$ is defined by

$$
A_{u ; g}(i, j)= \begin{cases}1 & \text { if } g^{-1}\left(\operatorname{Int}\left(I_{i}\right)\right) \cap \operatorname{Int}\left(I_{j}\right) \neq \varnothing, \quad 1 \leq i, j \leq N(\mathcal{U}) . \\ 0 & \text { otherwise, }\end{cases}
$$

4. Key lemmas for Theorem A. In this section we will assume that $f \in$ $C^{1}(X, X)$ satisfies the following conditions:

4-1. (i) the set of asymptotically stable periodic points for $f$ is finite (an empty set is allowed, but then there exists at least one absorbing boundary point).

(ii) $f$ is expanding on the complement of the union of the domains of attraction $A_{\infty}(f)$.

(iii) $U_{\mathrm{Bd}(X)}$ is an open neighborhood of the boundary of $X$ which consists of two components of the union of the domains of attraction of the asymptotically stable periodic points for $f$ and the absorbing boundary points of $X$ for $f$.

4-2. LEMMA. There exist positive integers $N$ and $M$ such that $\left|\left(f^{N}\right)^{\prime}(x)\right|>1$ for all $x \in A_{M}$.

ProOF. Let constants $c>0$ and $K>1$ be selected such that $\left|\left(f^{n}\right)^{\prime}(x)\right| \geq c \cdot K^{n}$ for all $x \in A_{\infty}(f)$, for each $n \in \mathbf{N}$. Let $N$ be a positive integer satisfying $c \cdot K^{N}>1$; assume that $N$ is minimal. We write $U_{N}$ for an open neighborhood of $A_{\infty}(f)$ such that $\left|\left(f^{N}\right)^{\prime}(x)\right|>1$ for all $x \in U_{N}$. Choose the positive integer $M$ minimally such that $A_{M} \subset U_{N}$, which exists because the $A_{k}$ form, from a certain nonnegative integer, a decreasing sequence of compact sets. 
4-3. LEMMA. For any nonnegative integer $n$ there exist positive integers $N\left(D_{n}\right)$ and $N\left(A_{n}\right)$, and there exists a nonnegative integer $T\left(A_{n}\right)$ such that the set $D_{n}$ consists of $N\left(D_{n}\right)$ components and the set $A_{n}$ consists of the union of $N\left(A_{n}\right)$ nontrivial components and $T\left(A_{n}\right)$ trivial components (points).

ProOF. By applying 4-2 fix positive integers $N$ and $M$ such that $\left|\left(f^{N}\right)^{\prime}(x)\right|>1$ for all $x \in A_{M}$. We write $U=\left\{D ; D\right.$ a component of $\bigsqcup_{i=1}^{M-1} D_{i}, D$ contains at least one critical point of $f\}$. The set $U$ consists of finitely many components of the set $\bigsqcup_{i=0}^{M-1} D_{i}$, since Crit $(f) \cap X \backslash U_{\mathrm{Bd}(X)}$ is a compact and nonempty set. Let $N(U)$ denote the number of elements of $U$.

Given any nonnegative integer $n$, we denote by $N\left(D_{0}\right)$ the number of components of the set $D_{0}$. Recall that $D_{0}$ has finitely many components and that $f$ is a monotone mapping on the components of $X \backslash U$. From $f\left(D_{n+1}\right) \subset f(X)$ and $D_{n+1}=\left\{x \in A_{n+1} ; f^{n+1}(x) \in D_{0}\right\}=\left\{x \in A_{n} ; f(x) \in A_{n}, f^{n}(f(x)) \in D_{0}\right\}$ we get $f\left(D_{n+1}\right)=D_{n} \cap f(x)$. Using this result one obtains that $(N(U)+1)^{n} \cdot N\left(D_{0}\right)$ is an upper bound for the number of components of the set $D_{n}$. Consequently, the set $A_{n}$ has finitely many components.

4-4. LEMMA. The number $m, m=\min \left\{n \in \mathbf{N} \cup\{0\} ; f^{n}(C r i t(f)) \subset D_{0}\right\}$, is well defined.

ProOF. Apply 4-1(iii), 4-2, and 2-2.

Now we will construct an open set $C$ such that $f \in \mathfrak{A}(C)$, with $\mathfrak{A}(C)$ the class of maps considered in $\S 3$. Let integer $m$ be as in 4-4. Let $D_{0}^{*}$ be a closed set in $X$, which is contained in the union of the direct domains of attraction $D_{0}$, with the following properties:

4-5. (i) each component of $D_{0}$ contains exactly one component of $D_{0}^{*}$, consequently the number of components of the set $D_{0}^{*}$ is equal to the number of components of the set $D_{0}$.

(ii) $f\left(D_{0}^{*}\right) \subset \operatorname{Int}\left(D_{0}^{*}\right)$, i.e. $D_{0}^{*}$ will be mapped into its interior under the map $f$.

(iii) If the set of asymptotically stable periodic points of $f$ is not empty, then it is contained in the interior of $D_{0}^{*}$.

(iv) $f^{m}(\operatorname{Crit}(f)) \subset \operatorname{Int}\left(D_{0}^{*}\right)$, i.e. the map $f^{m}$ maps the set of critical points of $f$ into the interior of $D_{0}^{*}$.

(v) If there is an absorbing boundary point $q$ then one of the components of $D_{0}^{*}$ is a neighborhood of $q$.

We define $B=\left\{x \in \bigsqcup_{i=0}^{m} D_{i} ; f^{m}(x) \in D_{0}^{*}\right\}$, and further we define $C=X \backslash B$. The set $C$ is open in $X$, since $B$ is closed in $X$. We write $\hat{D}_{0}^{*}=\{x \in D ; D$ a component of $D_{0}^{*}, D$ contains an asymptotically stable periodic point for $\left.f\right\}$. Note that the set $\hat{D}_{0}^{*}$ is empty in the case that $f$ has no asymptotically stable periodically points.

4-6. LEMMA. $f \in \mathfrak{A}(C)$, with $\mathfrak{A}(C)$ the class of mappings introduced in $\S 3$.

Proof. (i) From the definition of $B$, we have $f(B) \subset \operatorname{Int}(B)$; consequently $f^{-1}(C) \subset C$.

(ii) From $f(\operatorname{Crit}(f)) \subset \operatorname{Int}(B)$ it follows that $\operatorname{Crit}(f) \cap f^{-1}(C)=\varnothing$. Hence, the restriction of $f$ to the set $f^{-1}(C)$ is locally a diffeomorphism. 
(iii) From 4-5, 2-1, and the definition of $C$ we obtain: $\operatorname{Card}\{Y ; Y$ a component of $\left.f^{-1}(C)\right\}=\operatorname{Card}\left\{Y ; Y\right.$ a component of $\left.A_{m+2}\right\}$. By 4-3 we know that $A_{m+2}$ has finitely many components.

4-7. Lemma. Card $\left\{x \in C ; f^{n}(x)=x\right\}=\operatorname{Tr}\left(A_{C ; f^{n}}\right)$ for each $n \in \mathbf{N}$.

ProOF. We write $C=\bigsqcup_{i=1}^{N(C)} C_{i}$. Let a positive integer $n$ be fixed. Choose an integer $i, 1 \leq i \leq N(C)$.

Let $D$ denote a component of $f^{-n}\left(C_{i}\right) \cap C_{i}$. From the fact that the mapping $f^{n}$ is continuous, and $f^{n}(D)=C_{i}$, we have by applying the intermediate value theorem: the function $f^{n}$ has at least one fixed point in $D$. By the assumption that $f$ is expanding on $A_{\infty}(f)$ we obtain that the function $f^{n}$ has precisely one fixed point in $D$. Since the integer $i$ and the component $D$ are chosen arbitrarily, we get the result that the number of fixed points of the mapping $f^{n}$ in $C$ is equal to the trace of the matrix $A_{C ; f^{n}}$.

\section{4-8. LEMMA. f has a Markov partition.}

PROOF. The union of the components of the set $\hat{D}_{0}^{*} \cup \mathrm{Cl}(C)$ is a Markov partition.

We write $\left(\hat{D}_{0}^{*} \cup \mathrm{Cl}(C)\right) \cap f^{-1}\left(\hat{D}_{0}^{*} \cup \mathrm{Cl}(C)\right)=\bigcup_{k=1}^{N} I_{k}$ for some positive integer $N$. We define the matrix $A_{f}$ by

$$
A_{f}(i, j)= \begin{cases}1 & \text { if } f^{-1}\left(\operatorname{Int}\left(I_{i}\right)\right) \cap \operatorname{Int}\left(I_{j}\right) \neq \varnothing, \quad 1 \leq i, j \leq N . \\ 0 & \text { otherwise, }\end{cases}
$$

We denote by $N(\operatorname{Per}(f ; n))$ the number of periodic points for $f$ with period $n$, i.e. $N(\operatorname{Per}(f ; n))=\operatorname{Card}\left\{x \in X ; f^{n}(x)=x\right\}$, for every $n \in \mathbf{N}$. We set $\left\{\lambda_{k} ; 1 \leq k \leq H\right\}$ for the set of different eigenvalues of $A_{f}$ for some positive integer $H$ with $H \leq N$. Let $m_{k}$ denote the multiplicity of $\lambda_{k}, 1 \leq k \leq H$.

4-10. LEMMA. $N(\operatorname{Per}(f ; n))=\sum_{k=1}^{H} m_{k} \lambda_{k}^{n}$ for each $n \in \mathbf{N}$.

PROOF. Apply 4-1, 4-7, and (4-9).

4-11. LEMMA. $\zeta_{f}(z)=\prod_{k=1}^{H}\left(1-\lambda_{k} z\right)^{-m_{k}}$.

PrOOF. By applying 4-10 and the definition of $A_{f}$ we obtain

$$
\begin{aligned}
\exp \left[\sum_{n=1}^{\infty} \frac{N(\operatorname{Per}(f ; n))}{n} z^{n}\right] & =\prod_{k=1}^{H}\left[\exp \left[\sum_{n=1}^{\infty} \frac{\left(\lambda_{k} z\right)^{n}}{n}\right]\right]^{m_{k}} \\
& =\prod_{k=1}^{H}\left[\exp \left(-\log \left(1-\lambda_{k} z\right)\right)\right]^{m_{k}} \\
& =\prod_{k=1}^{H}\left[\frac{1}{1-\lambda_{k} \cdot z}\right]^{m_{k}} \cdot
\end{aligned}
$$

5. Key lemmas for Theorem $B$. In this section we will assume that the chaotic map $f \in C^{1}(X, X)$ satisfies the following conditions:

5-1. (i) $f \in C^{3}(X, X)$ and $f$ has a negative Schwarzian derivative, i.e. $S f(x)<0$ for all $x$ with $f^{\prime}(x) \neq 0$. 
(ii) The complement of the union of the domains of attraction $A_{\infty}(f)$ is compact and it is contained in the interior of $X$.

(iii) $\operatorname{Crit}(f) \subset \bigcup_{k=0}^{\infty} D_{k}$, i.e. the set of critical points of $f$ are contained in the union of the domains of attraction.

(iv) $f$ has no one-sided asymptotically stable periodic points.

5-2. LEMMA. The set consisting of the union of the asymptotically stable periodic points for $f$ and the absorbing boundary points is finite.

Proof. We write $s=\min \left\{x \in X ; x \in A_{\infty}(f)\right\}$ and $t=\max \{x \in X ; x \in$ $\left.A_{\infty}(f)\right\}$. The union of the components of $\bigcup_{k=0}^{\infty} D_{k}$ is an open cover of the nonempty compact set $\operatorname{Crit}(f) \cap[s, t]$. There are, say $n$, components from this cover covering $\operatorname{Crit}(f) \cap[s, t]$.

By a slight generalization of Singer's theorem [15] we know that the restriction of $f$ to $[s, t]$ has at most $n$ asymptotically stable periodic orbits (see [12]).

We conclude that the set of asymptotically stable periodic points for $f$ and aborbing boundary points is finite.

5-3. LEMMA. There exists a nonnegative integer $P$ such that for each $k \in \mathbf{N}$ we have: $A_{k+P}$ is compact and $\operatorname{Crit}\left(f^{k}\right) \cap A_{k+P}=\varnothing$.

PROOF. Let $s$ and $t$ be as in the proof of the preceding lemma. By the assumption $A_{\infty}(f) \subset \operatorname{Int}(X)$ we have that $X \backslash[s, t]$ is contained in $\bigcup_{k=0}^{\infty} D_{k}$. Since $\operatorname{Crit}(f) \cap[s, t]$ is compact we can find a nonnegative integer $P$ such that $\operatorname{Crit}(f) \cup X \backslash[s, t]$ is contained in $\bigcup_{k=0}^{P} D_{k}$. Assume that $P$ is minimal. Then $A_{1+P}$ is compact and $\operatorname{Crit}(f) \cap A_{1+P}=\varnothing$. By definition we have for each $k \in \mathbf{N}$ the set $A_{k+P}$ is compact. By induction we obtain $\operatorname{Crit}\left(f^{k}\right) \cap A_{k+P}=\varnothing$ for each $k \in \mathbf{N}$.

The following lemma is due to Misiurewicz [10].

5-4. LEMMA. Let $I$ be a closed interval. Denote by $U$ and $V$ two open subsets of $I$, consisting of a finite number of intervals each, for which $U$ contains the endpoints of $I$ and $U \cup V=I$. Let $g \in C^{3}(V, I)$ be given. Assume that $g$ satisfies the following conditions: (i) $g^{\prime}(x) \neq 0$ for all $x \in V$, (ii) $S g(x)<0$ for all $x \in V$, and (iii) $g$ has neither asymptotically stable periodic points nor one-sided asymptotically stable periodic points.

Then we have: there exists a positive integer $m$ such that, if $g^{j}(x) \notin U$ for all $j, 0 \leq j \leq m-1$, then $\left|\left(g^{m}\right)^{\prime}(x)\right|>1$.

ProOF. See the proof of Theorem 1.3 in Misiurewicz's paper [10].

5-5. LEMMA. There exists a positive integer $N$ such that $\left|\left(f^{N}\right)^{\prime}(x)\right|>1$ for all $x \in A_{N+P}$, with $P$ as in Lemma 5-3.

ProOF. Let $I \subset X$ be a compact interval such that $A_{\infty}(f) \subset \operatorname{Int}(I)$. Let integer $P$ be as in 5-3. We define $U=\bigcup_{k=0}^{P} D_{k} \cap I$. Let $V$ an open neighborhood of $A_{1+P}$ such that $\operatorname{Crit}(f) \cap V=\varnothing$ and $V$ does not contain (one-sided) asymptotically stable periodic points for $f$. By applying Lemma 5-4 we have that there exists a positive integer $N$ such that, if $f^{j}(x) \notin U$ for $0 \leq j \leq N-1$, then $\left|\left(f^{N}\right)^{\prime}(x)\right|>1$ for $x \in V$. Consequently $\left|\left(f^{N}\right)^{\prime}(x)\right|>1$ for all $x \in A_{N+P}$. 
5-6. LEMMA. $f$ is expanding on $A_{\infty}(f)$.

ProOF. Assume that $\left|\left(f^{N}\right)^{\prime}(x)\right|>1$ for all $x \in A_{N+P}$, with $P$ as in 5-3, and $N$ as in 5-5. We write $c=\min \left\{\left|f^{\prime}(x)\right| ; x \in A_{N+P}\right\}$, and $K=\min \left\{\left|\left(f^{N}\right)^{\prime}(x)\right| ; x \in\right.$ $\left.A_{N+P}\right\}$. Note that $c>0$ and $K>1$. If $c>1$ it is obvious that $g$ is an expanding map on $A_{\infty}(f)$. So from now on we assume $c \leq 1$.

Let a positive integer $n$ be given. We write $n=s N+t$ for some nonnegative integers $s, t$ with $0 \leq t \leq N-1$. Recalling that $A_{\infty}(f)$ is a positively $f$-invariant set, we have for each $x \in A_{\infty}(f)$ :

$$
\left|\left(f^{n}\right)^{\prime}(x)\right| \geq K^{s} c^{t} \geq\left[K^{1 / N}\right]^{s N} \cdot c^{N} \geq\left[K^{1 / N}\right]^{n} \cdot[c / K]^{n} .
$$

We conclude: $f$ is an expanding map on $A_{\infty}(f)$.

\section{Proofs of the results.}

ProOF OF THEOREM A. We assume that $X \subset \mathbf{R}$ is a nontrivial interval. Let $f \in C^{1}(X, X)$ be a chaotic mapping, and assume that $f$ satisfies conditions (1) and (2) of the theorem.

For each $n \in \mathbf{N}$ we have that $A_{\infty}(f) \subset A_{n}$. We write $s=\min \{x \in X ; x \in$ $\left.A_{\infty}(f)\right\}, t=\max \left\{x \in X ; x \in A_{\infty}(f)\right\}$. Since $f$ is a chaotic mapping, we have that $s<t$ and $\operatorname{Crit}(f) \cap[s, t] \neq \varnothing$.

The set consisting of asymptotically stable periodic points and absorbing boundary points is finite, since there are at most two absorbing boundary points and, if the set of asymptotically stable periodic points for $f$ is not empty then this set is finite because it is discrete and it is assumed to be compact.

We consider the following cases:
(a) $\operatorname{Bd}(X) \cap A_{\infty}(f)=\varnothing$;
(b) $\operatorname{Bd}(X) \cap A_{\infty}(f)=\{s\}$;
(c) $\operatorname{Bd}(X) \cap A_{\infty}(f)=\{t\}$;
(d) $\operatorname{Bd}(X) \cap A_{\infty}(f)=\{s, t\}$.

Case (a). Assume that $\operatorname{Bd}(X) \cap A_{\infty}(f)=\varnothing$. Then we have $A_{\infty}(f) \subset \operatorname{Int}(X)$. Applying 4-1, 4-8, (4-9), and 4-10 we obtain result (i); result (ii) follows from 4-11.

Case (b). Assume that $\operatorname{Bd}(X) \cap A_{\infty}(f)=\{s\}$. We define $u_{1}=\min \{x \in$ $[s, t] ; x \in \operatorname{Crit}(f)\}, u_{2}=\min \left\{f(x) ; u_{1} \leq x \leq t\right\}$, and, if there exist asymptotically stable periodic points for $f$, we define $u_{3}=\min \{x \in[s, t] ; x$ is an asymptotically stable periodic point for $f$; further we write $u_{m}=\min \left\{u_{1}, u_{2}, u_{3}\right\}$. Note that $u_{m}>s$ and that $f\left(u_{m}\right) \geq u_{m}$. The set $Y$, defined by $Y=\left\{x \in X ; x \geq u_{m}\right\}$ is a positively $f$-invariant set. Furthermore, we have that the set $A_{\infty}(f) \cap Y$ is a subset of $\operatorname{Int}(Y)$. Applying case (a) we obtain a matrix $A_{f \mid Y}$ for $f$ restricted to $Y$. Since the restriction of $f$ to $X \backslash Y$ is a homeomorphism without asymptotically stable periodic points, it follows that $X \backslash Y$ contains at most one fixed point of $f$. Obviously, one obtains $A_{f}$ by extending the matrix $A_{f \mid Y}$ in an appropriate manner.

Case (c). Assume that $\operatorname{Bd}(X) \cap A_{\infty}(f)=\{t\}$. We define $v_{1}=\max \{x \in$ $[s, t] ; x \in \operatorname{Crit}(f)\}, v_{2}=\max \left\{f(x) ; s \leq x \leq v_{1}\right\}$, and if the set of asymptotically stable periodic points for $f$ is not empty, we define $v_{3}=\max \{x \in[s, t] ; x$ is an asymptotically stable periodic point for $f\}$; further we write $v_{M}=\max \left\{v_{1}, v_{2}, v_{3}\right\}$. Note that $v_{M}<t$ and that $f\left(v_{M}\right) \leq v_{M}$. The set $Y$, defined by $Y=\{x \in X ; x \leq$ $\left.v_{M}\right\}$, is a positively $f$-invariant set. Similar to case (b) we obtain the desired results.

Case (d). Assume that $\operatorname{Bd}(X) \cap A_{\infty}(f)=\{s, t\}$. We define $u_{m}$ as in case (b), and we define $v_{M}$ as in case (c). We write $Y=\left[u_{m}, v_{M}\right]$. The set $Y$ is a positively 
$f$-invariant set and $A_{\infty}(f) \cap Y$ is contained in the interior of $Y$. By applying case (a) we obtain a matrix $A_{f \mid Y}$ that can be associated to the restriction of $f$ to $Y$. Since $X \backslash Y$ does not contain asymptotically stable periodic points for $f$, and since the restriction of $f$ to $X \backslash Y$ is a homeomorphism, it follows that $X \backslash Y$ contains at most either two unstable fixed points of $f$ or one unstable periodic orbit of period two. The matrix $A_{f}$ is obtained by extending the matrix $A_{f \mid Y}$ in an appropiate manner.

ProOF OF ThEOREM B. We assume that $X \subset \mathbf{R}$ is a nontrivial interval. Let $f \in C^{3}(X, X)$ be a chaotic mapping and assume that $f$ satisfies conditions (1) and (2) of the theorem. We write $s=\min \left\{x \in X ; x \in A_{\infty}(f)\right\}, t=\max \{x \in X ; x \in$ $\left.A_{\infty}(f)\right\}$.

We consider the cases:

(a) $\operatorname{Bd}(X) \cap A_{\infty}(f)=\varnothing$;

(b) $\operatorname{Bd}(X) \cap A_{\infty}(f)=\{s\}$;

(c) $\operatorname{Bd}(X) \cap A_{\infty}(f)=\{t\}$;

(d) $\operatorname{Bd}(X) \cap A_{\infty}(f)=\{s, t\}$.

First, we assume that $f$ has no one-sided asymptotically stable periodic points.

Case (a). Assume that $A_{\infty}(f) \cap \operatorname{Bd}(X)=\varnothing$; then we have $A_{\infty}(f) \subset \operatorname{Int}(X)$. Hence, there exist $n \in \mathbf{N}$ such that $A_{n} \subset \operatorname{Int}(X)$. Applying 5-1, 5-2, and 5-6 we obtain that $f$ satisfies conditions (1) and (2) of Theorem $\mathrm{A}$, and we are done.

Cases (b), (c), and (d) are similar to the cases of Theorem A.

Now we assume that $f$ has $L$ periodic orbits consisting of one-sided asymptotically stable points for some positive integer $L$. We denote by $p$ the smallest common multiple of the periods of the one-sided asymptotically stable periodic points for $f$.

Let $g: I \times X \rightarrow X$ be a one-parameter family of $C^{3}$-maps with negative Schwarzian derivative such that the following conditions hold: There is a parameter value $\lambda^{*}$ with the properties $g\left(\lambda^{*}, \cdot\right)=f$, either

$$
\frac{\partial g^{p}}{\partial \lambda}\left(\lambda^{*}, q\right)<0<\frac{\partial^{2} g^{p}}{\partial x^{2}}\left(\lambda^{*}, q\right) \quad \text { or } \quad \frac{\partial^{2} g^{p}}{\partial x^{2}}\left(\lambda^{*}, q\right)<0<\frac{\partial g^{p}}{\partial \lambda}\left(\lambda^{*}, q\right)
$$

for every one-sided asymptotically stable periodic point $q$ for $f$.

We need the following lemma. Let $G: I \times X \rightarrow X$ be a one-parameter family of $C^{2}$-maps. Assume that there is a point $x^{*}$, a parameter value $\lambda^{*}$ and a positive integer $n$ such that (1) $G^{n}\left(\lambda^{*}, x^{*}\right)=x^{*},(2)\left(\partial G^{n} / \partial x\right)\left(\lambda^{*}, x^{*}\right)=1$, (3) either $\left(\partial G^{n} / \partial \lambda\right)\left(\lambda^{*}, x^{*}\right)<0<\left(\partial^{2} G^{n} / \partial x^{2}\right)\left(\lambda^{*}, x^{*}\right)$ or $\left(\partial^{2} G^{n} / \partial x^{2}\right)\left(\lambda^{*}, x^{*}\right)<0<$ $\left(\partial G^{n} / \partial \lambda\right)\left(\lambda^{*}, x^{*}\right)$. Then there exist positive numbers $\delta_{1}, \delta_{2}$, and $\varepsilon$ such that the following properties hold:

(i) if $\lambda \in] \lambda^{*}-\delta_{1}, \lambda^{*}\left[\right.$ then $G^{n}(\lambda, \cdot)$ has no fixed points in $] x^{*}-\varepsilon, x^{*}+\varepsilon[$;

(ii) if $\lambda \in] \lambda^{*}, \lambda+\delta_{2}\left[\right.$ then $G^{n}$ has two fixed points in $] x^{*}-\varepsilon, x^{*}+\varepsilon[$, one is asymptotically stable and one is unstable. (For a proof see Guckenheimer [6]; see also Whitley [19].)

Assume that the positive numbers $\delta$ and $\varepsilon$ have been chosen such that for each $\lambda$ in $] \lambda^{*}, \lambda^{*}+\delta\left[\right.$ the map $g^{p}(\lambda, \cdot)$ has two fixed points in $] q-\varepsilon, q+\varepsilon$ [ for every one-sided asymptotically stable periodic point $q$ for $f$. Fix some $\lambda$ with $\lambda^{*}<\lambda<\lambda^{*}+\delta$; and we write $g$ for $g(\lambda, \cdot)$. Obviously, for any positive integer $n$, the number of periodic points for $g$ with period $n$ is equal to the number of periodic points for $f$ with period $n$ plus the number of one-sided asymptotically stable periodic points for $f$ 
with a divisor of $n$ as smallest period. Now it is easy to see that $\zeta_{f}$ is a rational function, but let us formulate it more precisely.

Denote the periodic orbits consisting of one-sided asymptotically stable periodic points for $f$ with $P_{i}, 1 \leq i \leq L$; for each $q \in P_{i}$ we write $p_{i}$ for the smallest period, $1 \leq i \leq L$. From

$$
N(\operatorname{Per}(g ; n))=N(\operatorname{Per}(f ; n))+\sum_{\substack{P_{i} \mid n \\ i=1}}^{L} p_{i}
$$

it follows that

$$
\begin{aligned}
\varsigma_{f}(z) & =\exp \left[\sum_{n=1}^{\infty} \frac{N(\operatorname{Per}(f ; n))}{n} z^{n}\right] \\
& =\exp \left[\sum_{n=1}^{\infty} \frac{N(\operatorname{Per}(g ; n))}{n} z^{n}\right] / \exp \left[\sum_{n=1}^{\infty} \frac{1}{n} \sum_{\substack{P_{i} \mid n \\
i=1}}^{L} p_{i} z^{n}\right] \\
& =\zeta_{g}(z) \cdot \prod_{i=1}^{L}\left(1-z^{p_{i}}\right) .
\end{aligned}
$$

Recalling that $\zeta_{g}$ is rational we conclude $\zeta_{f}$ is rational.

The matrix $A_{f}$ will be obtained by deleting the rows and columns of the ma$\operatorname{trix} A_{g}$ which are associated with the asymptotically stable periodic points of $g$ bifurcating from the one-sided asymptotically stable periodic points for $f$.

PROOF OF COROLlARY B2. Let $X \subset \mathbf{R}$ be a nontrivial compact interval. Fix any polynomial mapping $f: X \rightarrow X$ of degree $N+1$ for some $N \in \mathrm{N}$ such that each critical point of $f$ is real. Assume that the orbit of every critical point converges to an asymptotically stable periodic orbit of $f$. Then $f^{\prime}(x)=\gamma \prod_{k=1}^{N}\left(x-c_{k}\right)$, with $\gamma$ a nonzero real number and all the numbers $c_{k}, 1 \leq k \leq N$, real. For $x \in X \backslash\left\{c_{k} ; 1 \leq k \leq N\right\}$ we have (see also Collet and Eckmann [5]):

$$
\frac{f^{\prime \prime \prime}(x)}{f^{\prime}(x)}-\frac{3}{2}\left[\frac{f^{\prime \prime}(x)}{f^{\prime}(x)}\right]^{2}=-\sum_{\substack{k, j=1 \\ k<j}}^{N} \frac{1}{\left(x-c_{k}\right)\left(x-c_{j}\right)}-\frac{3}{2} \sum_{k=1}^{N}\left[\frac{1}{x-c_{k}}\right]^{2}<0 .
$$

We conclude that $f$ satisfies the conditions of Theorem B.

\section{REFERENCES}

1. D. J. Allwright, Hypergraphic functions and bifurcations in recurrence relations, SIAM J. Appl. Math. 34 (1978), 687-691.

2. M. Artin and B. Mazur, On periodic points, Ann. of Math. (2) 81 (1965), 82-99.

3. L. Block, Simple periodic orbits of mappings of the interval, Trans. Amer. Math. Soc. 254 (1979), 391-398.

4. R. Bowen, Markov partitions for Axiom A diffeomorphisms, Amer. J. Math. 92 (1970), 725-747.

5. P. Collet and J.-P. Eckmann, Iterated maps on the interval as dynamical systems, Birkhäuser, 1980.

6. J. Guckenheimer, On the bifurcations of maps of the interval, Invent. Math. 39 (1977), 165-178.

7. T. Y. Li and J. A. Yorke, Period three implies chaos, Amer. Math. Monthly 82 (1975), 985992. 
8. J. Milnor and W. Thurston, On iterated maps of the interval. I and II, Mimeographed, Princeton Univ., 1977.

9. M. Misiurewicz, Structure of mappings of an interval with zero entropy, Inst. Hautes Études Sci. Publ. Math. 53 (1981), 5-16.

10. _ Absolutely continuous measures for certain maps of an interval, Inst. Hautes Études Sci. Publ. Math. 53 (1981), 17-51.

11. Z. Nitecki, Topological dynamics on the interval, Ergodic Theory and Dynamical Systems. II, Progress in Math., 21, Birkhäuser, 1982, pp. 1-73.

12. H. E. Nusse, Chaos, yet no chance to get lost, Thesis, R. U. Utrecht, 1983.

13. C. Preston, Iterates of maps on an interval., Lecture Notes in Math., vol. 999, Springer-Verlag, 1983.

14. A. N. Sharkovsky, Coexistence of the cycles of a continuous mapping of the line into itself, Ukrain. Math. Zh. 16 (1964), 61-71.

15. D. Singer, Stable orbits and bifurcations of maps of the interval, SIAM J. Appl. Math. 35 (1978), 260-267.

16. S. Smale and R. F. Williams, The qualitative analysis of a difference equation of population growth, J. Math. Biol. 3 (1976), 1-4.

17. P. Stefan, $A$ theorem of Sharkovsky on the existence of periodic orbits of continuous endomorphisms of the real line, Comm. Math. Phys. 54 (1977), 237-248.

18. P. D. Straffin, Periodic points of continuous functions, Math. Mag. 51 (178), 99-105.

19. D. Whitley, Discrete dynamical systems in dimensions one and two, Bull. London Math. Soc. 15 (1983), 177-217.

Rijksuniversiteit Groningen, FaCUlteit der ECONOMische Wetenschappen, P. O. BOX 800, NL-9700, AV Groningen, The NetherlandS

Current address: University of Maryland, Institute for Physical Science and Technology, College Park, Maryland 20742 